\title{
Integration of traditional Chinese medicine and nibble debridement and dressing method reduces thrombosis and inflammatory response in the treatment of thromboangiitis obliterans
}

\author{
Jianhua $\mathrm{Li}^{1}$, Jingfeng Zhong ${ }^{1}$, Chunfa Huang ${ }^{1}$, Jiewen Guo ${ }^{2}$, Bingyu Wang ${ }^{2}$ \\ ${ }^{1}$ Vasculitis Department, Guangzhou Hospital of Traditional Chinese Medicine, Guangzhou, China; ${ }^{2}$ Science and Education Section, Guangzhou \\ Hospital of Traditional Chinese Medicine, Guangzhou, China \\ Contributions: (I) Conception and design: J Li; (II) Administrative support: J Li; (III) Provision of study materials or patients: J Li, J Zhong; (IV) \\ Collection and assembly of data: J Li, C Huang; (V) Data analysis and interpretation: J Guo, B Wang; (VI) Manuscript writing: All authors; (VII) \\ Final approval of manuscript: All authors. \\ Correspondence to: Dr. Jianhua Li. Vasculitis Department, Guangzhou Hospital of Traditional Chinese Medicine, No. 16 Zhuji Road, Liwan District, \\ Guangzhou 510130, China. Email: 1jh730512@126.com.
}

Background: Thromboangiitis obliterans (TAO), also known as Buerger's disease, is an occlusive arterial disease; however, the pathogenesis of TAO is still unclear. Research has shown that traditional Chinese medicine (TCM) has significant advantages in the treatment of TAO. Our purpose was to explore the underlying roles of TCM in combination with nibble debridement and dressing method (NDDM) in a TAO rat model.

Methods: We administered rats with $10 \mathrm{mg} / \mathrm{mL}$ sodium laurate to establish a TAO model, and then the TAO model rats were treated with notoginseng powder (NP), maifusheng (MFS), or the combination of NP or MFS and NDDM. Gangrene classification and blood rheology were evaluated; the pathological characteristics of rat limbs were examined by hematoxylin and eosin (H\&E) staining and Masson staining; and cluster of differentiation $3+(C D 3+)$ and cluster of differentiation 20+ (CD20+) levels were measured by immunohistochemistry (IHC) and flow cytometry. In addition, inflammation-associated cytokines were analyzed by quantitative reverse transcription polymerase chain reaction (RT-qPCR), western blot, and enzyme-linked immunosorbent assay (ELISA).

Results: Integration of NP or MFS and NDDM dramatically reduced the gangrene classification and affected blood rheology parameters of TAO model rats compared with NP and MFS alone. Meanwhile, NP or MFS in combination with NDDM decreased CD3+CD20+ T cells, reduced thrombosis and inflammatory cell infiltration, and dramatically decreased the levels of inflammation-associated cytokines.

Conclusions: Our results suggested that integration of NP or MFS and NDDM could relieve the symptoms of TAO model rats induced by sodium laurate, which might provide a new management strategy for TAO.

Keywords: Thromboangiitis obliterans (TAO); notoginseng powder (NP); maifusheng (MFS); nibble debridement and dressing method (NDDM); inflammation-associated cytokines

Submitted Jun 02, 2021. Accepted for publication Sep 03, 2021.

doi: $10.21037 / \mathrm{atm}-21-3752$

View this article at: https://dx.doi.org/10.21037/atm-21-3752 


\section{Introduction}

Thromboangiitis obliterans (TAO) is a chronic occlusive disease characterized by segmental, non-suppurative inflammation, and intravascular thrombus formation (1). It mainly invades the limbs, especially the small and medium arteries and veins of the lower limbs, and then causes ischemic changes in the distal side of the affected limb (2). At present, TAO mainly presents as limb ischemia, pain, intermittent claudication, migrating phlebitis, extremity gangrene, secondary infection, and even disability $(3,4)$. Studies have indicated that the pathogenesis of TAO is associated with a number of risk factors, such as smoking, infection, immune response, genetic inheritance, antiphospholipid antibody, hyperhomocysteinemia, cold, trauma, malnutrition, and so on (5). The current treatment, on the one hand, can alleviate the vascular damage caused by inflammation through anti-inflammation; on the other hand, it can reduce the response activity of pro-inflammatory immune cells through immunosuppression, so as to reduce the level of systemic inflammation, including application of vasodilators, improvement of microcirculation, antibiotics, glucocorticoids, traditional Chinese medicine (TCM), and surgical or interventional surgery, among others (6). However, the treatment of TAO is ineffective and recurrence is common. Although the short-term curative effect of surgical treatment is good, surgery can only improve blood circulation in the limbs to a degree and cannot change the process of arterial autoimmune response, so re-occlusion will occur with the extension of postoperative time. Therefore, it is urgent to explore effective therapies for TAO.

Notoginseng powder (NP) is a representative medicine of blood circulation drugs (7). Modern pharmacology studies have indicated that NP has multiple clinical effects, such as hemostasis (small dosage), anticoagulation (large dosage), blood tonic (long-term medication), suppression of inflammatory cells, cholesterol-lowering, treatment of arrhythmia, inhibition of tissue and organ fibrosis, tumor adjuvant treatment, and prevention of aging, among others (8). An important pharmacological property of NP that its long-term moderate oral administration does not incur any drug toxicity (9). However, the underlying therapeutic effect and mechanism of NP on TAO remain largely undetermined.

Maifusheng (MFS), as a Chinese herbal decoction, is often used to treat arteriosclerosis obliterans (10). Among the ingredients of MFS, beautiful Millettia root (Niu Da Li), prepared rhizome of Rehmannia (Shu Di Huang), and Caulis Spatholobi (Ji Xue Teng) have the effects of reinforcing kidney, nourishing the blood, and promoting blood circulation; Oldenlandia (Bai Hua She She Cao), Anemone raddeana Regel (Zhu Jie Xian Fu), and Shinyleaf Prickly-ash root (Liang Mian Zhen) have the effects of clearing away heat and toxic materials, expelling wind-damp, and activating the collaterals (11). Modern pharmacological studies have also confirmed that Caulis Spatholobi can reduce blood lipid, promote total phosphorus metabolism in kidney, and resist atherosclerosis; Oldenlandia has the functions of immunoregulation, anti-tumor, anti-bacteria, and anti-oxidation; the triterpenoid saponins in Anemone raddeana Regel have anti-tumor, anti-inflammation, antirheumatism, antipyretic analgesia, sedation, anti-convulsion effects (12). However, the therapeutic effect of MFS on TAO remains unclear.

Research has increasingly suggested that nibble debridement and dressing method (NDDM) can effectively promote ulcer surface healing (13). Studies have indicated that NDDM in combination with TCM could significantly improve the syndrome of diabetic foot ulcer (14). However, the effect of integrating NP or MFS and NDDM for the treatment of TAO has not yet been elucidated.

In this study, sodium laurate was used to induce a TAO rat model, and the mechanism and therapeutic effects of NP or MFS in combination with NDDM on the symptoms of TAO were further investigated. We present the following article in accordance with the ARRIVE reporting checklist (available at https://dx.doi.org/10.21037/atm-21-3752).

\section{Methods}

\section{Animals}

A total of 48 healthy male Wistar rats were provided by the Guangzhou Hospital of Traditional Chinese Medicine, Guangzhou (Guangdong, China). According to the national standard, all rats were raised for 7 days before the experiment, under the following conditions: access to food and water ad libitum, temperature of $20-24^{\circ} \mathrm{C}$, humidity of 50-60\%, lights on from 8:00 AM to 8:00 PM. All animal protocols were approved by the Animal Ethics Committee of Guangzhou Hospital of Traditional Chinese Medicine (No.: 2015NK023), in compliance with Guangzhou Hospital of Traditional Chinese Medicine guidelines for the care and use of animals.

\section{Establishment of TAO model}

As described in previous research (15), 48 male Wistar rats 
were randomly allocated to two groups: sham group $(\mathrm{n}=8)$ and TAO model group $(n=40)$. The rats were anesthetized by intraperitoneal injection of $1 \%$ pentobarbital sodium (50 mg/kg, Sigma-Aldrich, St. Louis, MO, USA, Cat\# P-010). The rats were then fixed in the supine position, and the inner femur of the left lower limb was shorn and skinned to expose the femoral artery. Blood flow was blocked by artery clipping, the femoral artery in the sham group was injected with $0.1 \mathrm{~mL}$ normal saline, and the femoral artery in the TAO model group was injected with $0.1 \mathrm{~mL}$ sodium laurate solution $(10 \mathrm{mg} / \mathrm{mL})$. After 20 mins of injection, the artery clamp was removed and the wound was stitched up.

\section{Grouping}

The rats were divided into a sham group $(\mathrm{n}=8)$, TAO group $(\mathrm{n}=8)$, TAO + NP group $(\mathrm{n}=8)$, TAO + MFS group $(\mathrm{n}=8), \mathrm{TAO}+\mathrm{NP}+\mathrm{NDDM}$ group $(\mathrm{n}=8)$, and TAO + MFS + NDDM group $(n=8)$. The different groups received the following: sham group (healthy rats, administered saline alone, $2 \mathrm{~mL} /$ day for $7 \mathrm{~d}$ ); TAO group (TAO model rats, administered saline alone, $2 \mathrm{~mL} /$ day for $7 \mathrm{~d}$ ); NP group (TAO model rats, NP, $2 \mathrm{~mL} /$ day for $7 \mathrm{~d}$, lavage administration; the ulcer was washed and bandaged with $0.2 \%$ Anerdian); MFS group (TAO model rats, MFS, $4 \mathrm{~mL} /$ day for $7 \mathrm{~d}$, lavage administration; the ulcer was washed and bandaged with $0.2 \%$ Anerdian); NP + NDDM group (TAO model rats, NP, $2 \mathrm{~mL} /$ day for $7 \mathrm{~d}$, lavage administration; the ulcer was treated by NDDM); MFS + NDDM group (TAO model rats, MFS, $4 \mathrm{~mL} /$ day for $7 \mathrm{~d}$, lavage administration; the ulcer was treated by NDDM). Rats were fed and observed for 14 days after gavage, blood was collected from the orbits on days 1, 7, and 14 , and partial serum was isolated on the 14 th day. The experimental rats were put to death in a $\mathrm{CO}_{2}$ euthanasia chamber, all rats were euthanized and their inner hind leg muscles were collected on day 14 . The tissues were fixed with $4 \%$ paraformaldehyde (Merck KGaA, Darmstadt, Germany, Cat. \#1.04005).

\section{Classification of gangrene}

According to the grading standard for gangrene, level 0 : normal; level I, gangrene confined to the toenail; level II: gangrene confined to the toes; level IIII gangrene confined to the foot; level IV, gangrene exceeds the ankle joint (16). The classification of gangrene was conducted at 3 time points (1, 7, and 14 days) after intragastric administration.

\section{Hemorbeology detection}

Blood samples were collected from the eye socket of rats on day 1,7 , and 14 after intragastric administration, and put into ethylenediaminetetraacetic acid (EDTA)-containing Vacutainers (BD Biosciences, Becton, Dickinson, and Co., Franklin Lakes, NJ, USA), respectively. Blood platelet count (BPC), white blood cell count (WBC), fibrinogen, plasma viscosity (PV), red blood cell count (RBC), hemoglobin $(\mathrm{Hb})$, neutrophil counts $(\mathrm{NC})$, and erythrocyte sedimentation rate (ESR) levels were measured using an automatic biochemistry analyzer (Roche, Basel, Switzerland cobas c 311) and hemorheology system (HLIFE, Shandong, China, LB-2A).

\section{$R N A$ extraction and real-time quantitative polymerase chain reaction assay}

Total RNAs were extracted from the muscles on the inside of the right hind limbs using TRIzol reagent (\#9109, Takara Bio., Shiga, Japan). The quality of total RNAs was examined using NanoDro2000c (Thermo Scientific, Waltham, MA, USA). We used $2 \mu \mathrm{g}$ RNAs to synthesize circular DNA (cDNA) using the reverse transcription kit (Takara). The polymerase chain reaction (PCR) amplification was performed using SYBR GREEN PCR Master Mix (Applied Biosystems, Forster City, CA, USA). The results were counted by $2^{-\Delta \Delta \mathrm{Ct}}$ method (17). The sequences of primers are shown in Table 1.

\section{Western blot assay}

The muscles on the inside of the right hind limbs were added to a little liquid nitrogen and quickly crushed. Proteins were extracted using radioimmunoprecipitation assay (RIPA) buffer (Beyotime, Shanghai, China, Cat. No. $\mathrm{P} 0013 \mathrm{~B}$ ) including phenylmethylsulfonyl fluoride (PMSF) (Genebase, Bethesda, MD, USA, \#329-98-6/\#115-39-9). A bicinchoninic acid (BCA) kit (Thermo Scientific) was utilized to determine the concentrations of proteins. Total proteins $(30 \mu \mathrm{g})$ were separated using $10 \%$ sodium dodecyl sulfate polyacrylamide gel electrophoresis (SDSPAGE), transferred onto nitrocellulose membranes (Millipore, Burlington, MA, USA). After blocking with $5 \%$ skimmed milk (BD Biosciences), the membranes were incubated with primary antibodies overnight at $4{ }^{\circ} \mathrm{C}$. The next day, the membranes were incubated with horseradish peroxidase (HRP) goat anti-rabbit IgG 
Table 1 The sequences of primers in this study

\begin{tabular}{llc}
\hline ID & Sequence (5'-3') & $\begin{array}{c}\text { Product length } \\
(\mathrm{bp})\end{array}$ \\
\hline GAPDH F & CCTCGTCTCATAGACAAGATGGT & 169 \\
GAPDH R & GGGTAGAGTCATACTGGAACATG & \\
TNF-a F & CTTCTCATTCCTGCTCGTGG & 201 \\
TNF-a R & TCCGCTTGGTGGTTTGCTA & \\
IL-1 F & TTTGAGTCTGCACAGTTCCC \\
IL-1 R & AACTATGTCCCGACCATTGC & 96 \\
IL-6 F & ACCACCCACAACAGACCAGTA & 232 \\
IL-6 R & CAGAGCAGATTTTCAATAGGCA & \\
IL-17 F & CCTCAGACTACCTCAACCGT & 142 \\
IL-17 R & ATGTGGTGGTCCAACTTCCC & \\
IFN- $\gamma$ F & GTCATCGAATCGCACCTGAT \\
IFN- $\gamma$ R & GGATCTGTGGGTTGTTCACC & \\
\hline
\end{tabular}

(1:20,000, Boster Bio., Wuhan, China, cat\# BA1054) or HRP goat anti-mouse IgG (1:20,000, Boster Bio., cat\# BA1051) for $40 \mathrm{~min}$. Finally, the membranes were treated with the enhanced chemiluminescent reagents (Millipore, WBKLS0500), the results were observed by X-ray films (XBT-1, Eastman Kodak Company, Rochester, NY, USA), and the data were analyzed by Image-Pro Plus 6.0 (Media Cybernetics, Inc., Rockville, MD, USA). The primary antibodies included interleukin-17 (IL-17) (1:1,000, Abcam, Cambridge, UK, ab77171), interferon- $\gamma$ (IFN- $\gamma)(1: 1,000$, Abcam, ab77246), IL-1 $\beta$ (1:1,000, Abcam, ab2105), IL-6 (1:1,000, Abcam, ab208113), tumor necrosis factor- $\alpha(\mathrm{TNF}-\alpha)(1: 1,000$, Abcam, ab6671) and glyceraldehyde 3-phosphate dehydrogenase (GAPDH) (1:2,000, Abcam, ab9482).

\section{Enzyme-linked immunosorbent assay detection}

After 14 days, serum was isolated from the blood of all rats. According to the manufacturer's protocols, the concentrations of IL-17, IFN- $\gamma$, IL- $\beta$, IL- 6 , and TNF- $\alpha$ were measured using commercial enzyme-linked immunosorbent assay (ELISA) kits as per the instructions of manufacturers. The ELISA kits included IL-1 $\beta$ (Cusabio, Houston, TX, USA, CSB-E08055r), IL-6 (Cusabio, CSBE04640r), IL-17 (Cusabio, CSB-E07451r), IFN- $\gamma$ (Cusabio,
CSB-E04579r), and TNF- $\alpha$ (Cusabio, CSB-E11987r).

\section{Immunobistochemistry assay}

The samples were embedded with paraffin, and cut into $4 \mu \mathrm{m}$ section. After dewaxing and hydration, the antigen retrieval was performed with sodium citrate buffer at $95^{\circ} \mathrm{C}$. The sections were treated with $3 \%$ hydrogen peroxide for $10 \mathrm{~min}$. Then, the sections were blocked with $3 \%$ bovine serum albumin (BSA) at $37^{\circ} \mathrm{C}$ for $30 \mathrm{~min}$ and incubated with anti-CD3 (1:150, Abcam, ab16669) and anti-CD20 (1:400, Bioss, Woburn, MA, bs-20639R) overnight at $4{ }^{\circ} \mathrm{C}$. The following day, the sections were incubated with goat antirabbit IgG H\&L (HRP, 1:1,000, Abcam, ab6721) for $50 \mathrm{~min}$ at room temperature. The sections were then treated with diaminobenzidine (DAB) for $5 \mathrm{~min}$, hematoxylin for $3 \mathrm{~min}$, and $1 \%$ acid alcohol for a few seconds. Finally, the sections were observed under a light microscope (Olympus, CX-21).

\section{Haematoxylin and eosin staining}

The medial muscles of the right hind limbs were fixed with $4 \%$ paraformaldehyde and dehydrated with gradient alcohol. Then, $4 \mu \mathrm{m}$ sections were stained with hematoxylin and eosin (H\&E) solution (H8070, Solarbio, Beijing, China). The pathology results were obtained under a microscope (Eclipse Ci, Nikon, Tokyo, Japan).

\section{Masson staining}

The sections were treated with dimethylbenzene and dehydrated with gradient ethanol. Then, the sections were stained using Masson staining kit (G1006). After staining, the sections were treated with $1 \%$ glacial acetic acid for $1 \mathrm{~min}$. Finally, the sections were treated with graded ethanol and dimethylbenzene. The results were obtained using a microscope (Nikon Eclipse Ci).

\section{Statistical analysis}

The results were calculated using the software GraphPad Prism version 7 (GraphPad Prism Software, La Jolla, CA, USA). All data were expressed as mean \pm standard deviation (SD). Differences between groups were analyzed by oneway analysis of variance (ANOVA) or Student's $t$-test. A P value $<0.05$ was considered significant. 


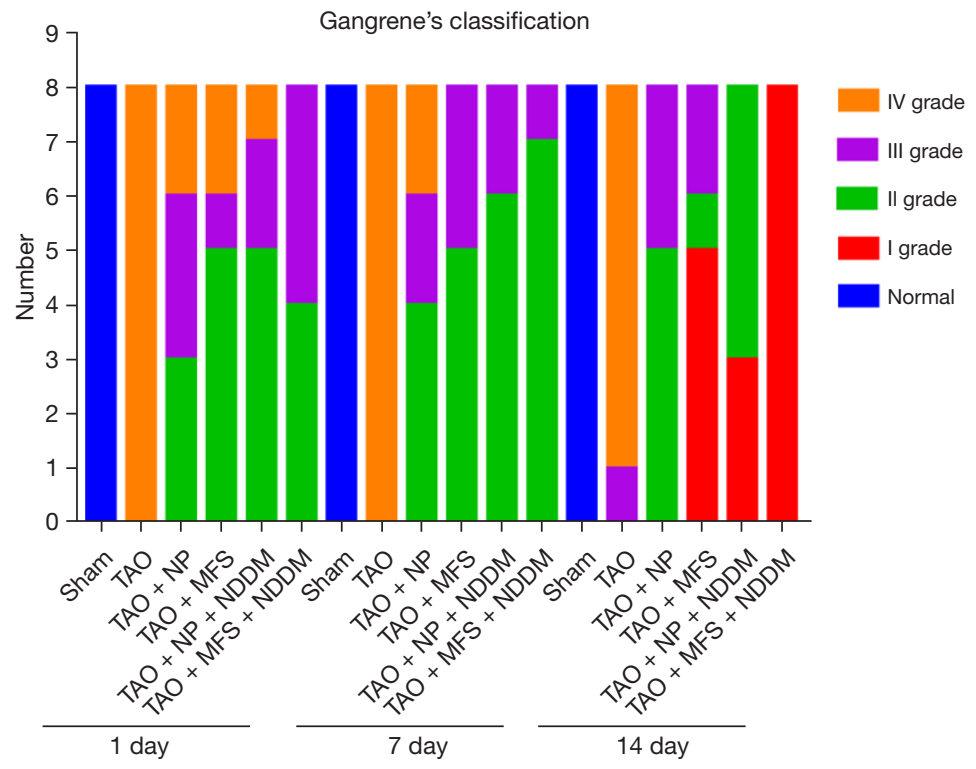

Figure 1 Effects of NP, MFS, or NDDM on the gangrene classification grade of TAO rats. TAO model rats were administered NP or MFS for 7 days, and ulcers were treated with NDDM. The extent of the gangrene was ranked by the Grade Evaluation of Gangrene on the 1st, 7th, and 14th day. TAO, thromboangiitis obliterans; NP, notoginseng powder; MFS, maifusheng; NDDM, nibble debridement and dressing method.

\section{Results}

\section{The combination of NP or MFS and NDDM reduced the gangrene classification level of TAO rats}

To investigate the role of integrating TCM and NDDM in the treatment of TAO, TAO model rats were treated with NP, MFS, or the combination of NP or MFS and NDDM. The results of gangrene classification revealed that rats in the sham group showed normal performance; pathological changes of the limbs of TAO model rats were all concentrated in grade IV gangrene; pathological changes of rats in the NP or MFS group were mainly concentrated in grade II and III gangrene; and pathological changes of rats in the NP or MFS plus NDDM group were mainly concentrated in grade I and II gangrene. Overall, the manifestations of rats in the TAO model group all were grade IV gangrene, and the gangrene were obviously reduced in the NP or MFS group compared with the TAO model group, the gangrenes were also dramatically reduced in the NP or MFS in combination with NDDM groups compared with the NP or MFS alone group. In addition, our results revealed that along with the increase of the drug treatment time, the gangrene was gradually relieved, especially, the combined treatment group and the single treatment group on the 14th day after intragastric administration (Figure 1).

\section{The impacts of NP or MFS in combination with NDDM on bemorbeology index}

Next, we examined the effects of NP or MFS in combination with NDDM treatments on hemorheology indexes. The results indicated that $\mathrm{BPC}, \mathrm{RBC}, \mathrm{WBC}$, fibrinogen, and $\mathrm{PV}$ levels were significantly increased in the TAO model group relative to the sham group, and NP and MFS alone or in combination with NDDM could decrease BPC, RBC, WBC, fibrinogen, and PV levels. While, Hb, NC, and ESR levels were not affected by NP, MFS, or either in combination with NDDM (Table 2).

\section{NP or MFS in combination with NDDM reduced thrombosis and inflammatory infiltration of rat limbs}

The pathological section of muscle tissues in the sham group showed smooth tunica interna, well-ranged muscle fibers, and no thrombus or inflammatory cell infiltration. The pathological section of muscle tissues in the medial side of the right hind limb in the TAO model group showed large amounts of thrombosis, disordered arrangement of muscle fibers, no atrophy, fibrosis, or calcification in tunica media, thickened tunica externa without fibrosis, and many infiltrating inflammatory cells. It was shown that NP or 
Table 2 Hematological and hemorheological parameters in Sham, TAO, NP, MFS, NP + NDDM, and MFS + NDDM rats

\begin{tabular}{|c|c|c|c|c|c|c|c|c|}
\hline Indicators & $\operatorname{BPC}\left(10^{8} / \mathrm{mL}\right)$ & $\operatorname{RBC}\left(10^{8} / \mathrm{mL}\right)$ & $\mathrm{Hb}(\mathrm{g} / \mathrm{L})$ & WBC $\left(10^{8} / \mathrm{mL}\right)$ & $\mathrm{NC}\left(10^{8} / \mathrm{mL}\right)$ & Fibrinogen (g/L) & $\mathrm{ESR}(\mathrm{mm} / \mathrm{h})$ & $\mathrm{PV}(\mathrm{mPa} \cdot \mathrm{s})$ \\
\hline 1 day & $7.15 \pm 0.12$ & $6.40 \pm 0.06$ & $125.64 \pm 3.83$ & $4.61 \pm 0.31$ & $1.06 \pm 0.03$ & $2.46 \pm 0.08$ & $1.02 \pm 0.04$ & $1.48 \pm 0.07$ \\
\hline 7 days & $7.2 \pm 0.09$ & $6.38 \pm 0.29$ & $126.90 \pm 5.24$ & $4.51 \pm 0.25$ & $1.09 \pm 0.05$ & $2.42 \pm 0.08$ & $1.08 \pm 0.04$ & $1.33 \pm 0.07$ \\
\hline 14 days & $7.15 \pm 0.08$ & $6.40 \pm 0.11$ & $131.39 \pm 10.01$ & $4.54 \pm 0.26$ & $1.07 \pm 0.08$ & $2.85 \pm 1.18$ & $1.08 \pm 0.04$ & $1.49 \pm 0.15$ \\
\hline 1 day & $8.97 \pm 0.92^{*}$ & $6.06 \pm 0.10^{\star \star}$ & $126.04 \pm 5.19$ & $5.08 \pm 0.06$ & $1.06 \pm 0.07$ & $2.77 \pm 0.13^{*}$ & $1.06 \pm 0.05$ & $2.46 \pm 0.31^{*}$ \\
\hline 7 days & $9.59 \pm 0.76^{*}$ & $5.83 \pm 0.13^{\star \star}$ & $115.52 \pm 6.97$ & $5.05 \pm 0.10^{*}$ & $1.12 \pm 0.07$ & $2.91 \pm 0.13^{\star}$ & $1.12 \pm 0.03$ & $2.66 \pm 0.32^{*}$ \\
\hline 14 days & $11.24 \pm 1.07^{\star}$ & $5.60 \pm 0.21^{\star \star}$ & $111.93 \pm 8.24$ & $5.23 \pm 0.06^{*}$ & $1.22 \pm 0.09$ & $3.06 \pm 0.11^{*}$ & $1.18 \pm 0.07$ & $3.55 \pm 0.79^{*}$ \\
\hline \multicolumn{9}{|l|}{ NP } \\
\hline \multicolumn{9}{|l|}{ MFS } \\
\hline 1 day & $9.06 \pm 1.07$ & $6.24 \pm 0.15$ & $128.45 \pm 4.16$ & $5.08 \pm 0.04$ & $1.05 \pm 0.06$ & $2.75 \pm 0.12$ & $1.04 \pm 0.07$ & $2.21 \pm 0.17^{\#}$ \\
\hline 7 days & $8.98 \pm 0.63^{\#}$ & $6.21 \pm 0.49$ & $119.95 \pm 7.58$ & $5.04 \pm 0.11$ & $1.10 \pm 0.05$ & $2.88 \pm 0.13$ & $1.10 \pm 0.04$ & $2.05 \pm 0.18^{\#}$ \\
\hline 14 days & $8.82 \pm 0.52^{\#}$ & $6.75 \pm 0.17^{\#}$ & $118.92 \pm 8.89$ & $5.15 \pm 0.09$ & $1.18 \pm 0.09$ & $2.80 \pm 0.10^{\#}$ & $1.15 \pm 0.05$ & $3.18 \pm 0.89^{\#}$ \\
\hline \multicolumn{9}{|c|}{$\mathrm{NP}+\mathrm{NDDM}$} \\
\hline 1 day & $8.55 \pm 0.56$ & $6.42 \pm 0.36$ & $126.19 \pm 12.77$ & $5.06 \pm 0.11$ & $1.10 \pm 0.13$ & $2.58 \pm 0.25$ & $1.05 \pm 0.13$ & $2.50 \pm 0.08^{\$}$ \\
\hline 7 days & $9.30 \pm 0.66$ & $6.14 \pm 0.37$ & $124.19 \pm 5.97$ & $5.08 \pm 0.10$ & $1.13 \pm 0.08$ & $2.55 \pm 0.15$ & $1.07 \pm 0.18$ & $2.15 \pm 0.16$ \\
\hline
\end{tabular}

Values were expressed as means $\pm \mathrm{SD}$. ${ }^{*} \mathrm{P}<0.05$, ${ }^{\star \star} \mathrm{P}<0.01$ vs. Sham group; ${ }^{\sharp} \mathrm{P}<0.05$ vs. TAO group; ${ }^{\$} \mathrm{P}<0.05$ vs. NP group; ${ }^{\mathrm{P}} \mathrm{P}<0.05$ vs. MFS group. BPC, blood platelet count; RBC, red blood cell count; Hb, hemoglobin; WBC, white blood cell count; NC, neutrophil counts; ESR, erythrocyte sedimentation rate; PV, plasma viscosity; TAO, thromboangiitis obliterans; NP, notoginseng powder; MFS, maifusheng; NDDM, nibble debridement and dressing method.

MFS treatment could improve the pathological histology of rat limbs, and NP or MFS in combination with NDDM further recovered the pathological histology of rat limbs (Figure $2 A$ ). In addition, Masson staining was performed to assess the degree of vascular fibrosis. Plentiful new blood vessels and few thrombi were observed in the sham group, while histopathology in the TAO model group indicated the accumulation of collagen fibers and obvious thrombus organization. We found that compared with the sham group, the degrees of collagen deposition and vascular fibrosis were increased in the TAO model group, and NP or MFS treatment markedly attenuated the degrees of collagen deposition and vascular fibrosis of rats in the TAO model group. The combination of NP or MFS with NDDM further decreased the degrees of collagen deposition and vascular fibrosis of rats in the TAO model group (Figure 2B).

\section{NP or MFS in combination with NDDM decreased CD3 $+C D 20+T$ cells}

To identify the changes of $\mathrm{CD} 3+\mathrm{CD} 20+\mathrm{T}$ cells in TAO model rats after NP or MFS in combination with NDDM, 


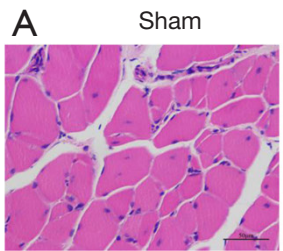

B

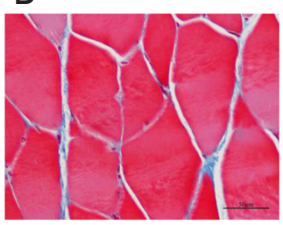

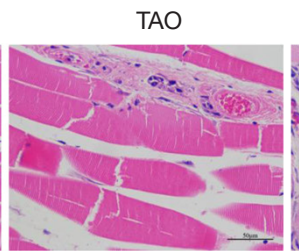

TAO

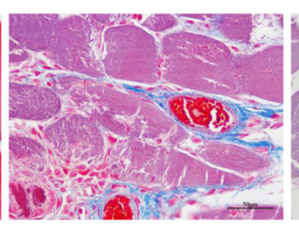

$\mathrm{TAO}+\mathrm{NP}$

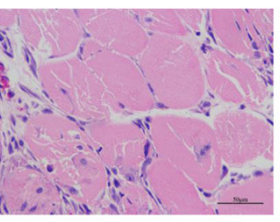

$\mathrm{TAO}+\mathrm{NP}$

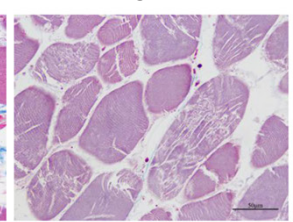

$\mathrm{TAO}+\mathrm{MFS}$

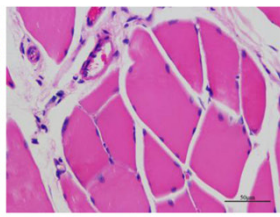

$\mathrm{TAO}+\mathrm{MFS}$

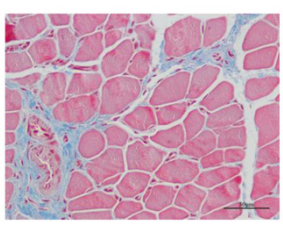

$\mathrm{TAO}+\mathrm{NP}+\mathrm{NDDM}$

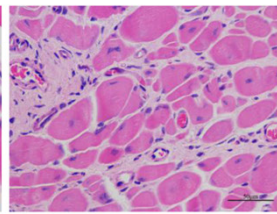

$\mathrm{TAO}+\mathrm{NP}+\mathrm{NDDM}$

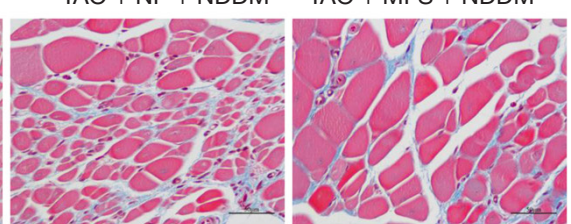

Figure 2 NP or MFS in combination with NDDM reduced thrombosis and inflammatory infiltration of rat limbs. TAO model rats which were administrated with NP or MFS, and ulcers were treated with NDDM. (A) The degree of vascular damage in rats was assessed by H\&E staining, magnification, $\times 400$; scale bar $=50 \mu \mathrm{m}$. (B) The degree of vascular fibrosis in rats was evaluated by Masson staining, magnification, $\times 400$; scale bar $=50 \mu \mathrm{m}$. TAO, thromboangiitis obliterans; NP, notoginseng powder; MFS, maifusheng; NDDM, nibble debridement and dressing method; H\&E, hematoxylin and eosin.

we determined CD3 and CD20 levels. The results from immunohistochemical (IHC) assay indicated that the expression of CD3 was dramatically decreased in the TAO model group relative to the sham group, markedly increased in the NP or MFS group compared with the TAO model group, and significantly increased in the NP or MFS in combination with NDDM groups in comparison to the NP or MFS alone group; meanwhile, CD20 expression was dramatically increased in the TAO model group relative to the sham group, markedly decreased in the NP or MFS group compared with the TAO model group, and significantly decreased in the NP or MFS in combination with NDDM groups in comparison to the NP or MFS alone group (Figure $3 A$ ). In addition, flow cytometry revealed that compared with sham group, CD3+CD20+ T cells were markedly increased in the TAO model group. Meanwhile, NP or MFS treatment decreased CD3+CD20+ $\mathrm{T}$ cells in TAO model group rats, and NP or MFS in combination with NDDM further decreased CD3+CD20+ T cells (Figure 3B).

\section{NP or MFS in combination with NDDM inbibited inflammation-associated cytokines}

To further explore the influence of NP or MFS in combination with NDDM on inflammation-associated cytokines in TAO model rats, quantitative reverse transcription PCR (RT-qPCR), western blot, and ELISA assays were utilized to analyze IL-17, IFN- $\gamma$, IL-1 $\beta$, IL-6, and TNF- $\alpha$ levels. As shown in Figure 4, IL-17, IFN- $\gamma$, IL-1 $\beta$, IL-6, and TNF- $\alpha$ levels were higher in the TAO model group than the sham group, and lower in the TAO model rats with NP or MFS treatment than the TAO model rats. The levels of IL-17, IFN- $\gamma$, IL-1 $\beta$, IL-6, and TNF- $\alpha$ expression were significantly decreased in the TAO model rats with NP or MFS in combination with NDDM treatments compared with NP or MFS treatment alone.

\section{Discussion}

Lauric acid is a free saturated fatty acid in serum (18). After a large amount of lauric acid is injected into the blood vessels, it accumulates in the corresponding blood vessels, which will cause vascular endothelial cells and vascular wall injury, induce thrombosis, cause local vascular circulation disorder, and eventually result in a localized ischemic state $(19,20)$. Lauric acid has previously been widely used to induce TAO model (21). Thus, we administered an injection of $0.1 \mathrm{~mL}$ sodium laurate solution $(10 \mathrm{mg} / \mathrm{mL})$ in the femoral artery of rats to achieve the TAO model in this study. According to classification of gangrene, rats in the sham group showed normal performance, rats in TAO model group were all concentrated in grade IV gangrene, suggesting that the TAO model had been successfully established. In addition, we demonstrated that treatment with NP or MFS alone could reduce the degree of limb injury in rats, and the injuries were subsequently mainly concentrated in gangrene grades II and III; the combination 

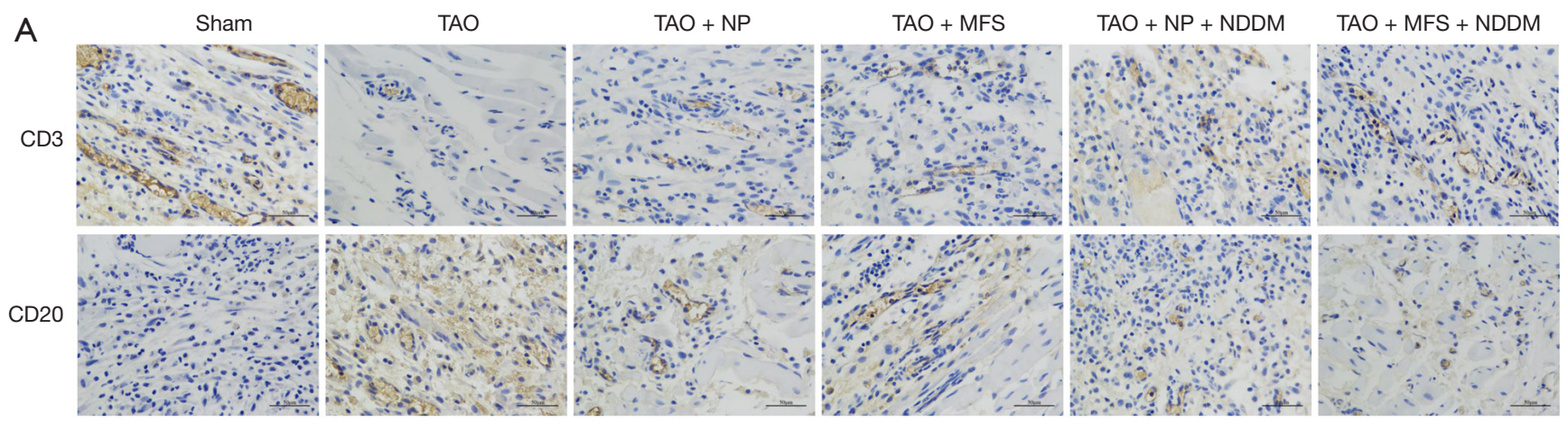

B
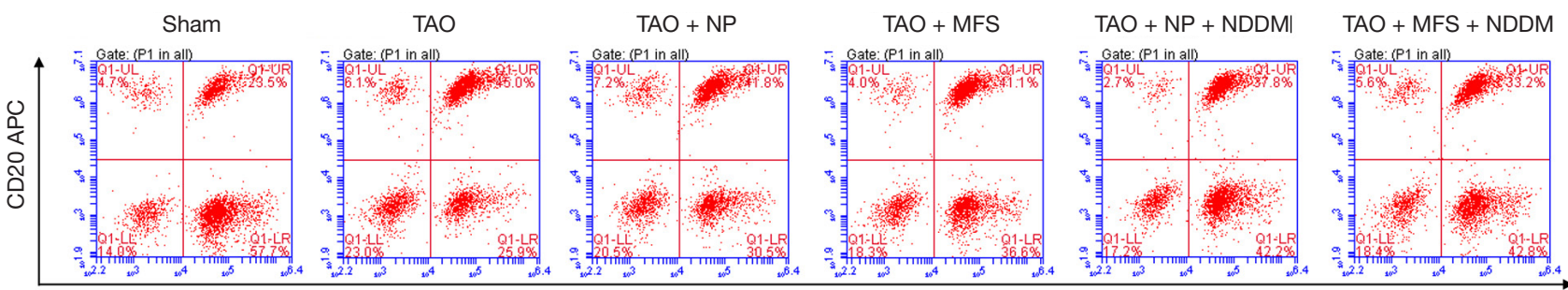

CD3 FITC

Figure 3 The influence of NP or MFS in combination with NDDM on CD3+CD20+ T cells. TAO model rats were treated with NP or MFS, and ulcers were treated with NDDM. (A) The CD3+ and CD20+ levels were examined by IHC assay in each group, respectively. Magnification, $\times 200$, scale bars $=50 \mu \mathrm{m}$. (B) Peripheral blood extracted from rats in each group was dyed with isotype controls, anti-CD20 and anti-CD3 antibodies, and the level of CD3+CD20+ T cells was analyzed by flow cytometry. TAO, thromboangiitis obliterans; NP, notoginseng powder; MFS, maifusheng; NDDM, nibble debridement and dressing method; IHC, immunohistochemistry.

of NP or MFS and NDDM could dramatically alleviate the degree of limb injury in rats, and the injuries were subsequently mainly concentrated in gangrene grades I and II. Therefore, we suggested that integration of NP or MFS and NDDM noticeably reduced the classification grade of gangrene of TAO rats.

Although TAO is among the most common chronic periarterial ischemic disorders in China, the etiology and pathogenesis have not yet been fully elucidated (21). To date, research has shown that autoimmune mechanisms, especially abnormal erythrocyte immune function and hemorheology changes, play essential roles in the occurrence and development of TAO (22). Study has shown that traditional Chinese medicine has a certain effect in relieving or treating TAO. Recently, study by Li et al. has shown that herbal therapy has a certain effect on relieving symptoms of TAO patients with severe limb pain (23). In addition, Salvianolic acid B also shows anti-inflammatory and anti-thrombotic effects in vivo, and can be used as a potential drug for the treatment of TAO (24). Studies have suggested that blood rheology is closely associated with a variety of diseases, such as chronic heart failure (25), sickle cell anemia (26), angina pectoris (27), diabetic retinopathy (28), and so on. Our research indicated that NP or MFS in combination with NDDM could decrease BPC, WBC, fibrinogen, and PV levels. Therefore, we deduced that integration of TCM and NDDM could affect blood rheology.

A human leukocyte differentiation antigen, CD20 is expressed on the surface of B cells at all stages of cell development and differentiation except plasma cells, and is recognized as the surface marker of B cells (29). As the co-differentiation antigen of mature $\mathrm{T}$ cells, CD3 is expressed on the surface of all mature $\mathrm{T}$ cells (30). Together, CD3 and $\mathrm{T}$ cell receptor (TCR) can form CD3TCR complex through salt bridge, which can transfer the antigen stimulation signal received by TCR into cells. Therefore, CD3 plays a crucial role in the process of $\mathrm{T}$ cell immunity (31). Prior studies have confirmed that a group of CD3 T cells also express CD20 molecules in the body apart from $\mathrm{B}$ cells, which have similar and unique biological characteristics to conventional CD3 $\mathrm{T}$ cells (26). It has been demonstrated that CD3+CD20+ T cells play an important role in multiple diseases (32), such as rheumatoid arthritis $(33,34)$, multiple sclerosis (32), psoriasis (35), lymphoproliferative disorder (36), and some 

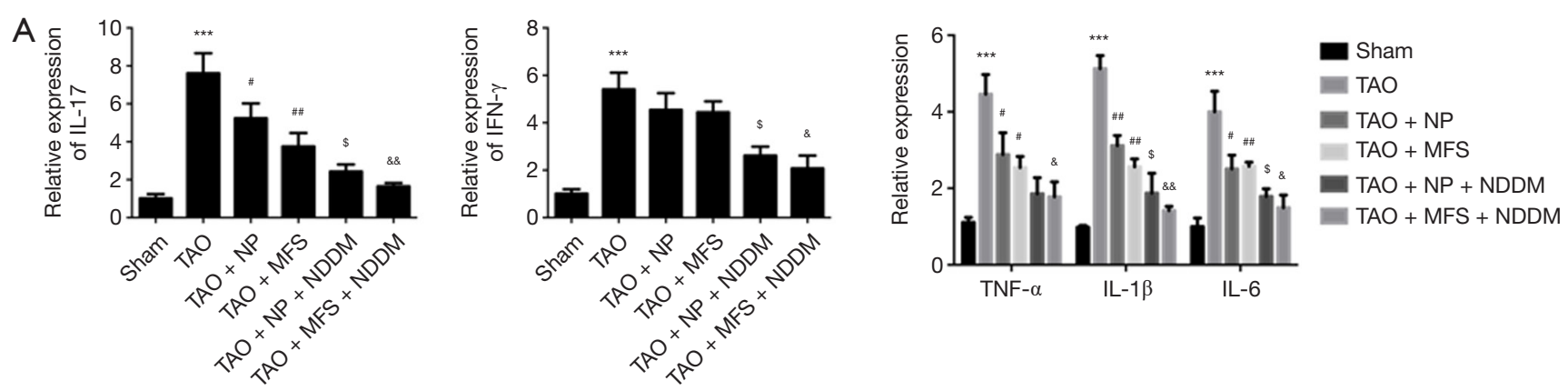

B
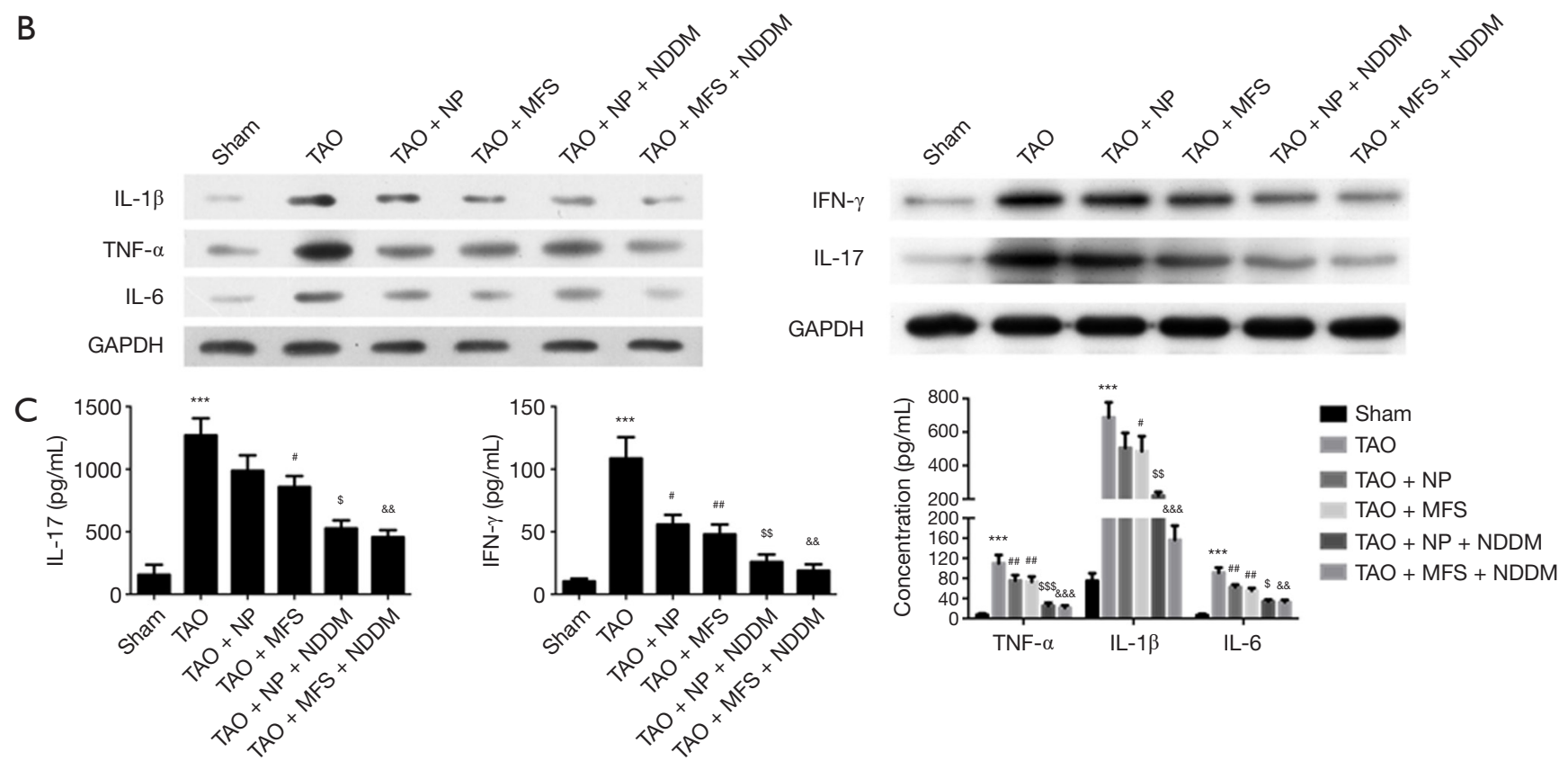

Figure 4 Effects of NP or MFS combination with NDDM on inflammation-associated cytokines. TAO model rats were administered with NP or MFS, and ulcers were treated with NDDM. (A) Expression levels of IL-17, IFN- $\gamma$, IL-1 $\beta$, IL- 6 , and TNF- $\alpha$ were assessed by RTqPCR assay in each group. (B) Western blot assay was carried out to examine IL-17, IFN- $\gamma$, IL-1 $\beta$, IL-6, and TNF- $\alpha$ expressions. GAPDH was used as a reference. (C) The relative concentrations of IL-17, IFN- $\gamma$, IL-1 $\beta$, IL-6, and TNF- $\alpha$ were analyzed by ELISA in the serum of rats in each group. ${ }^{* *} \mathrm{P}<0.001$ vs. the sham group; ${ }^{\#} \mathrm{P}<0.05,{ }^{\# \#} \mathrm{P}<0.01$ vs. the TAO group; ${ }^{\$} \mathrm{P}<0.05,{ }^{\$ S} \mathrm{P}<0.01,{ }^{\$ S S} \mathrm{P}<0.001$ vs. the TAO + NP group; ${ }^{\&} \mathrm{P}<0.05,{ }^{\& \&} \mathrm{P}<0.01,{ }^{\& \& \&} \mathrm{P}<0.001$ vs. the TAO + MFS group. TAO, thromboangiitis obliterans; NP, notoginseng powder; MFS, maifusheng; NDDM, nibble debridement and dressing method; RT-qPCR, quantitative reverse transcription polymerase chain reaction; ELISA, enzyme-linked immunosorbent assay; GAPDH, glyceraldehyde 3-phosphate dehydrogenase.

cancers $(31,32)$. In our study, we further confirmed that $\mathrm{NP}$ or MFS treatment decreased CD3+CD20+ T cells in TAO model rats, which could be further decreased via the synergy of NP or MFS combined with NDDM. In addition, we verified that NP or MFS in combination with NDDM treatment could improve thrombus and inflammatory cell infiltration of rat limbs, and decreased the degrees of collagen deposition and vascular fibrosis of rats in the TAO model group. Therefore, we further verified that the combination of NP or MFS and NDDM could relieve the symptoms of TAO.

Studies have shown that TAO, as an inflammatory disease, is associated with the inflammatory response of endothelial cells, platelets, leukocytes, and sensory neurons (37). Inflammation is significantly correlated with thrombogenesis, and thrombosis and vasculitis are major precursors of gangrene in many systemic inflammatory diseases, such as TAO, antineutrophil cytoplasmic antibody (ANCA)-related vasculitis, Takayasu's arteritis, systemic lupus erythematosus, antiphospholipid syndrome (APS), and 
inflammatory bowel disease, among others (32). Numerous studies have also shown that inflammation-associated cytokines, such as IL-1 $\beta$, IL-4, IL-17, IL-23, IL-6, and TNF- $\alpha$ are associated with TAO (32). The results of our study supported that NP or MFS in combination with NDDM dramatically decreased IFN- $\gamma$, IL- $1 \beta$, IL-6, and TNF- $\alpha$ levels, indicating that the combination of NP or MFS and NDDM inhibited inflammation-associated cytokines.

\section{Conclusions}

Our study demonstrated that NP or MFS combined with NDDM could reduce gangrene classification grade and change blood rheology of TAO rats. The combination NP or MFS and NDDM could improve thrombus, inflammatory cell infiltration, muscle fiber, and collagen fiber of rat limbs. Our findings also supported that the abovementioned combination could dramatically decrease $\mathrm{CD} 3+\mathrm{CD} 20+\mathrm{T}$ cells and inhibit inflammation-associated cytokines. Therefore, we suggest that the combination NP or MFS and NDDM might be a potential treatment for TAO. However, there are still some shortcomings in clinical use, such as complex dialectical classification, non-standard diagnosis, non-uniform curative effect evaluation, lack of in-depth clinical research, and so on, which still need to be further improved.

\section{Acknowledgments}

Funding: This study was financially supported by the Unilateral Prescription and TCM Characteristic Technology Arrangement Projects of Administration of Traditional Chinese Medicine of Guangdong Province (20164025) and Guangdong Scientific Research Project of Traditional Chinese Medicine (20201260). These funding bodies supported the study design and data collection.

\section{Footnote}

Reporting Checklist: The authors have completed the ARRIVE reporting checklist. Available at https://dx.doi. org/10.21037/atm-21-3752

Data Sharing Statement: Available at https://dx.doi. org/10.21037/atm-21-3752

Conflicts of Interest: All authors have completed the ICMJE uniform disclosure form (available at https://dx.doi. org/10.21037/atm-21-3752). The authors have no conflicts of interest to declare.

Ethical Statement: The authors are accountable for all aspects of the work in ensuring that questions related to the accuracy or integrity of any part of the work are appropriately investigated and resolved. All the animal protocols were approved by the Animal Ethics Committee of Guangzhou Hospital of Traditional Chinese Medicine (No.: 2015NK023), in compliance with Guangzhou Hospital of Traditional Chinese Medicine guidelines for the care and use of animals.

Open Access Statement: This is an Open Access article distributed in accordance with the Creative Commons Attribution-NonCommercial-NoDerivs 4.0 International License (CC BY-NC-ND 4.0), which permits the noncommercial replication and distribution of the article with the strict proviso that no changes or edits are made and the original work is properly cited (including links to both the formal publication through the relevant DOI and the license). See: https://creativecommons.org/licenses/by-nc-nd/4.0/.

\section{References}

1. Neufang A, Vargas-Gomez C, Ewald P, et al. Very distal vein bypass in patients with thromboangiitis obliterans. Vasa 2017;46:304-9.

2. Huang ZH, Kuo SY, Chiu YH, et al. Treatment of multiple refractory ankle ulcerations in thromboangiitis obliterans: A case report. Medicine (Baltimore) 2018;97:e10798.

3. Mills JL, Porter JM. Buerger's disease (thromboangiitis obliterans). Ann Vasc Surg 1991;5:570-2.

4. Kawarada O, Kume T, Ayabe S, et al. Endovascular Therapy Outcomes and Intravascular Ultrasound Findings in Thromboangiitis Obliterans (Buerger's Disease). J Endovasc Ther 2017;24:504-15.

5. Maslowski L, McBane R, Alexewicz P, et al. Antiphospholipid antibodies in thromboangiitis obliterans. Vasc Med 2002;7:259-64.

6. Sun XL, Law BY, de Seabra Rodrigues Dias IR, et al. Pathogenesis of thromboangiitis obliterans: Gene polymorphism and immunoregulation of human vascular endothelial cells. Atherosclerosis 2017;265:258-65.

7. Tang J, Gan S, Zheng M, et al. Efficacy of Endovascular Radiofrequency Ablation for Thromboangiitis Obliterans (Buerger's Disease). Ann Vasc Surg 2017;42:78-83. 
8. Chiang IH, Chen SG, Tzeng YS. Treatment of Thromboangiitis Obliterans Using Smoking Cessation and Far-infrared Therapy: A Case Study. Ostomy Wound Manage 2017;63:20-3.

9. Fazeli B, Dadgar Moghadam M, Niroumand S. How to Treat a Patient with Thromboangiitis Obliterans: A Systematic Review. Ann Vasc Surg 2018;49:219-28.

10. Martin-Rufino JD, Lozano FS, Redondo AM, et al. Sequential intravenous allogeneic mesenchymal stromal cells as a potential treatment for thromboangiitis obliterans (Buerger's disease). Stem Cell Res Ther 2018;9:150.

11. Dong JJ, Liu YJ, Tu JY, et al. Hypolipidemic effect of powder of Panax notoginseng and its mechanism. Chinese Traditional and Herbal Drugs 2017;48:1597-603.

12. Liu Y, Hao F, Zhang H, et al. Panax notoginseng saponins promote endothelial progenitor cell mobilization and attenuate atherosclerotic lesions in apolipoprotein $\mathrm{E}$ knockout mice. Cell Physiol Biochem 2013;32:814-26.

13. Zheng CS, Xu XJ, Ye HZ, et al. Computational pharmacological comparison of Salvia miltiorrhiza and Panax notoginseng used in the therapy of cardiovascular diseases. Exp Ther Med 2013;6:1163-8.

14. Du M. The affect of maifusheng on artery endothelial cell apoptosis. Modern Hospital 2006;6:18.

15. Lai ZH, Li WJ, Wang XR, et al. The Application Values of Clinical Analysis Report about Treatment of Arteriosclerotic Occlusive Disease by Maifusheng with MSCTA. Chinese Journal of CT and MRI 2012;3:71-4.

16. Wolff H, Hansson C. Larval therapy--an effective method of ulcer debridement. Clin Exp Dermatol 2003;28:134-7.

17. Leung PC. Diabetic foot ulcers--a comprehensive review. Surgeon 2007;5:219-31.

18. Waycaster C, Milne C. Economic and clinical benefit of collagenase ointment compared to a hydrogel dressing for pressure ulcer debridement in a long-term care setting. Wounds 2013;25:141-7.

19. Cuomo R, D'Aniello C, Grimaldi L, et al. EMLA and Lidocaine Spray: A Comparison for Surgical Debridement in Venous Leg Ulcers. Adv Wound Care (New Rochelle) 2015;4:358-61.

20. Xie XS, Wang YJ, Zuo C, et al. A case report of an effective treatment for diabetic foot ulcers with integration of traditional Chinese medicine and Western medicine. J Diabetes Complications 2009;23:360-4.

21. Liu C, Kong X, Wu X, et al. Alleviation of A disintegrin and metalloprotease 10 (ADAM10) on thromboangiitis obliterans involves the HMGB1/RAGE/ NF- $\kappa$ B pathway. Biochem Biophys Res Commun 2018;505:282-9.
22. Cacione DG, do Carmo Novaes F, Moreno DH. Stem cell therapy for treatment of thromboangiitis obliterans (Buerger's disease). Cochrane Database Syst Rev 2018;10:CD012794.

23. Li G, Zefr C, Yang B, et al. Herbal therapy treatment in thromboangiitis obliterans: a retrospective clinical study. Ann Palliat Med 2020;9:1696-707.

24. Zhang Z, Ji J, Zhang D, et al. Protective effects and potential mechanism of salvianolic acid B on sodium laurate-induced thromboangiitis obliterans in rats. Phytomedicine 2020;66:153110.

25. Tikhomirova I, Petrochenko E, Muravyov A, et al. Microcirculation and blood rheology abnormalities in chronic heart failure. Clin Hemorheol Microcirc 2017;65:383-91.

26. Lemonne N, Möckesch B, Charlot K, et al. Effects of hydroxyurea on blood rheology in sickle cell anemia: A two-years follow-up study. Clin Hemorheol Microcirc 2017;67:141-8.

27. Rivera-Chavarría IJ, Brenes-Gutiérrez JD. Thromboangiitis obliterans (Buerger's disease). Ann Med Surg (Lond) 2016;7:79-82.

28. Calanca L, Alatri A, Krieger C, et al. Thromboangiitis obliterans: notions for practice. Rev Med Suisse 2017;13:2129-33.

29. Melillo E, Grigoratos C, Sanctis FD, et al. Noninvasive Transcutaneous Monitoring in Long-Term Follow-Up of Patients With Thromboangiitis Obliterans Treated With Intravenous Iloprost. Angiology 2015;66:531-8.

30. Iwamoto A, Kajikawa M, Maruhashi T, et al. Vascular Function and Intima-media Thickness of a Leg Artery in Peripheral Artery Disease: A Comparison of Buerger Disease and Atherosclerotic Peripheral Artery Disease. J Atheroscler Thromb 2016;23:1261-9.

31. Van Caeneghem Y, De Munter S, Tieppo P, et al. Antigen receptor-redirected $\mathrm{T}$ cells derived from hematopoietic precursor cells lack expression of the endogenous TCR/ CD3 receptor and exhibit specific antitumor capacities. Oncoimmunology 2017;6:e1283460.

32. Schuh E, Berer K, Mulazzani M, et al. Features of Human CD3+CD20+ T Cells. J Immunol 2016;197:1111-7.

33. Liu H, Cai M. Effect of probucol on hemodynamics, rheology and blood lipid of diabetic retinopathy. Exp Ther Med 2018;15:3809-14.

34. Gelfand JM, Cree BAC, Hauser SL. Ocrelizumab and Other CD20+ B-Cell-Depleting Therapies in Multiple Sclerosis. Neurotherapeutics 2017;14:835-41.

35. Shanehbandi D, Majidi J, Kazemi T, et al. CD20- 
based Immunotherapy of B-cell Derived Hematologic Malignancies. Curr Cancer Drug Targets 2017;17:423-44.

36. Zapata DA, Schamel WW, Torres PS, et al. Biochemical differences in the alphabeta $\mathrm{T}$ cell receptor.CD3 surface complex between CD8+ and CD4+ human mature $\mathrm{T}$ lymphocytes. J Biol Chem 2004;279:24485-92.

Cite this article as: Li J, Zhong J, Huang C, Guo J, Wang B. Integration of traditional Chinese medicine and nibble debridement and dressing method reduces thrombosis and inflammatory response in the treatment of thromboangiitis obliterans. Ann Transl Med 2021;9(18):1413. doi: 10.21037/atm$21-3752$
37. Chen Q, Yuan S, Sun H, et al. CD3+CD20+ T cells and their roles in human diseases. Hum Immunol 2019;80:191-4.

(English Language Editor: J. Jones) 